\title{
Experiências vivenciadas em uma farmácia de manipulação: um relato de experiência
}

Experiences living in a manipulation pharmacy: a report of experience

Experiencias vividas en una farmacia de manipulación: un relato de experiencia

Francisco Patricio de ANDRADE JÚNIOR ${ }^{1}$

Júlia Beatriz Pereira de SOUZA²

${ }^{1}$ Doutorando, Programa de Pós-Graduação em Produtos Naturais e Sintéticos Bioativos, Universidade Federal da Paraiba (UFPB) João Pessoa-PB, Brasil

${ }^{2}$ Prof $^{a}$. Dr ${ }^{a}$. e Orientadora, Centro de Educação e Saúde, Universidade Federal de Campina Grande (UFCG) Cuité-PB, Brasil

\section{Resumo}

Introdução: A realização de estágios permite que o futuro profissional farmacêutico possa ter um contato mais precoce com situações cotidianas que ocorrem diariamente na jornada laboral. Ademais, esse tipo de experiência contribui para por em práticas conhecimentos teórico-práticos anteriormente adquiridos. Contudo, na literatura existem poucos estudos que relatem a experiência vivenciada em estágios, sobretudo, em farmácia magistral. Objetivo: Assim, o presente trabalho teve como objetivo relatar as experiências adquiridas durante o estágio em farmácia com manipulação. Materiais e métodos: Tratou-se de um estudo descritivo, do tipo relato de experiência realizado entre setembro a novembro de 2018. Resultados: Durante o estágio foi possível produzir cápsulas contendo hormônios, antimicrobianos e medicamentos em geral, assim como sachês, além de xampus, condicionadores, hidratantes, clareadores e algumas suspensões. Ainda ficava como encargo do estagiário fazer a calibração de todas as balanças, diariamente e de produzir o álcool a 70\% utilizado para a limpeza de bancadas, equipamentos e materiais. Conclusão: Assim, conclui-se que a experiência de realizar estágio em farmácia com manipulação pode contribuir para o desenvolvimento de novas habilidades e conhecimentos e possibilita que o estudante de farmácia se sinta mais seguro e preparado para atuar com a manipulação de medicamentos.

Descritores: Educação Farmacêutica; Estágios; Preparações Farmacêuticas.

\section{Abstract}

Introduction: Internships allow the future professional pharmacist to have an earlier contact with daily situations that occur in the workday. In addition, this type of experience contributes to practice previously acquired theoretical-practical knowledge. However, in the literature there are few studies that report the experience lived in stages, especially in magisterial pharmacy. Objective: The objective of this study was to report the experiences gained during the pharmacy internship with manipulation. Materials and methods: This was a descriptive study, a type of experience report carried out between September and November of the 2018. Results: During the stage it was possible to produce capsules containing hormones, antimicrobials and medications in general, as well as sachets, as well as shampoos, conditioners, moisturizers, bleaching agents and some suspensions. It was still up to the trainee to calibrate all the scales daily and produce the $70 \%$ alcohol used to clean benches, equipment and materials. Conclusion: Thus, it is concluded that the experience of performing a pharmacy internship with manipulation can contribute to the development of new skills and knowledge and enables the student of pharmacy to feel more secure and prepared to act with the manipulation of medicines.

Descriptors: Pharmaceutical Education; Traineeships; Pharmaceutical Preparations.

\section{Resumen}

Introducción: La realización de prácticas permite que el futuro profesional farmacéutico pueda tener un contacto más precoz con situaciones cotidianas que ocurren diariamente en la jornada laboral. Además, este tipo de experiencia contribuye a poner en práctica conocimientos teórico-prácticos anteriormente adquiridos. Sin embargo, en la literatura existen pocos estudios que relatan la experiencia vivida en la pasantía, sobre todo, en farmacia magistral. Objetivo: Así, el presente trabajo tuvo como objetivo relatar las experiencias adquiridas durante la pasantía en farmacia con manipulación. Materiales y métodos: Se trató de un estudio descriptivo, del tipo relato de experiencia realizado entre septiembre a noviembre de 2018. Resultados: Durante la etapa fue posible producir cápsulas conteniendo hormonas, antimicrobianos y medicamentos en general, así como sajés, champús, acondicionadores, hidratantes, blanqueadores y algunas suspensiones. Todavia el pasante era responsable por hacer la calibración de las balanzas a diario y de producir el alcohol al 70\% utilizado para la limpieza de encimeras, equipos y materiales. Conclusión: Así, se concluye que la experiencia de realizar pasantía en farmacia con manipulación puede contribuir al desarrollo de nuevas habilidades y conocimientos y posibilita que el estudiante de farmacia se sienta más seguro y preparado para actuar con la manipulación de medicamentos.

Descriptores: Educación Farmacéutica; Pasantías; Preparaciones Farmacéuticas

INTRODUÇÃO

No curso de bacharelado em farmácia da Universidade Federal de Campina Grande (UFCG) o graduando em farmácia deve realizar diversos estágios obrigatórios para obter o título de bacharel em farmácia. Dentre estes é possível destacar o Estágio Supervisionado III, que compreende produção de medicamentos por manipulação ou industrial ou alimentos, para o qual, o estudante deve optar por um único nicho.

O estágio deve apresentar carga-horária mínima de 300 horas, sendo que para cursar tal disciplina é necessário que o estudante tenha adquirido conhecimentos de química farmacêutica, farmacotécnica, tecnologia farmacêutica e de cosméticos, microbiologia, controle de qualidade físico-químico e biológico para medicamentos e cosméticos, biossegurança, deontologia farmacêutica, dentre outros ${ }^{1}$.

Assim, o farmacêutico que atua em farmácia magistral, mais especificamente, deve ter domínio acerca da legislação, documentação, estrutura, processos, gestão e orientação farmacêutica, para assim, permitir o desenvolvimento de produtos e serviços de qualidade ${ }^{2}$.

Além disso, é possível evidenciar que a farmácia com manipulação é um estabelecimento em que necessita-se da união de diversos conhecimentos, uma vez que, é um estabelecimento de saúde, no qual há a preparação magistral, ou seja, de medicamentos personalizados para cada um de seus usuários que estejam portando uma prescrição $0^{3,4}$.

Dessa forma, torna-se imprescindível que a farmácia siga os preceitos da Agência Nacional de Vigilância Sanitária (ANVISA) e que possua instalações e equipamentos adequados, assim como, pessoal qualificado para o desenvolvimento das atividades magistrais ${ }^{5}$ de forma que, é importante que o estudante de farmácia tenha um contato prévio com a manipulação, levando em consideração a necessidade de desenvolver conhecimentos teórico- 
práticos acerca das boas práticas de manipulação e registro de atividades, treinamentos e contato com processos burocráticos ${ }^{6}$ antes de terminar o curso de graduação.

Assim, levando em consideração a complexidade e o papel do farmacêutico como único profissional habilitado e responsável por gerenciar tudo aquilo que é produzido na farmácia magistral, percebe-se a relevância de relatar as experiências adquiridas durante o estágio, uma vez que, estas informações podem servir para embasar outros estudos que tenham a mesma temática como foco centralizador.

\section{MATERIAL E MÉTODO}

Estudo descritivo, do tipo relato de experiência ${ }^{7}$, realizado entre setembro a novembro de 2018 em uma farmácia de manipulação.

\section{RESULTADOS E DISCUSSÃO}

A farmácia com manipulação em que houve o desenvolvimento do estágio localizava-se no município de Campina Grande-PB e era dividida em zona limpa e zona suja, em que a zona suja é aquela em há a livre circulação de pessoas, levando, portanto, contaminantes ao ambiente, já a zona limpa, é aquela em que há restrição na entrada de pessoas e produtos, de forma que quando alguém adentra esse ambiente deve estar devidamente paramentado para evitar o carreamento de contaminantes, sendo portanto, de extrema importância essa divisão para garantir a qualidade, sobretudo microbiológica, dos produtos manipulados.

$\mathrm{Na}$ zona suja, tinha-se a recepção, hall, banheiro, uma sala administrativa, sala de paramentação, copa, depósito de material de limpeza e sala de lavagem de utensílios. Enquanto que na área limpa tinham-se um laboratório para a manipulação de semissólidos e líquidos e diversos laboratórios para a manipulação de sólidos, divididos em manipulação de antimicrobianos, citostáticos, hormônios e medicamentos em geral.

A farmácia magistral em que houve o desenvolvimento do estágio trabalhava com distintos grupos de substâncias, estando estas classificadas de acordo com a RDC 64/2007 em grupo I e III, sendo que os demais grupos não eram trabalhados. $\mathrm{O}$ grupo I corresponde a manipulação de medicamentos obtidos a partir de insumos/matérias primas, que pode ser, inclusive, de origem vegetal, enquanto que o grupo III refere-se aos antibióticos, citostáticos, hormônios e substâncias sujeitas a controle especial ${ }^{3}$.

A farmácia contava com um auxiliar de serviços gerais (ASG), duas recepcionistas, um técnico e uma farmacêutica, sendo esta última também a proprietária do estabelecimento. A jornada laboral iniciava-se $\operatorname{logo}$ após a paramentação utilizando-se alguns Equipamentos de Proteção Individual (EPIs) como luvas, máscara, touca e propé, sendo estes importantes para evitar não só a contaminação das preparações, mas também possível aquisição de doença ocupacional ${ }^{8}$. Além de ser necessário estar de calça comprida e não portar nenhum tipo de acessório como brincos, pulseiras, anéis e relógios.

- Sólidos

O setor de sólidos era composto por bancadas, armários, pias, balanças, exaustores e recipientes contendo insumos farmacêuticos e cápsulas. Neste setor, as atividades iniciavam-se a partir da avaliação da prescrição e juntamente com o técnico ou o farmacêutico havia-se o cálculo da quantidade de princípio ativo e excipientes que deveriam ser utilizados, assim como, o tamanho da cápsula ou sachê.

- Sachês

Na produção de sachês, o pó era geralmente misturado a algum flavorizante e, após a pesagem, este pó era adicionado ao interior do sachê, finalizando a etapa por meio da vedação a partir de uma máquina de prensa. Em seguida o sachê era adicionado a uma caixa e a mesma era identificada com os dados relacionados à farmácia, como contato, endereço, nome e CRF do responsável técnico, assim como, informações sobre o produto manipulado e do paciente.

○ Cápsulas

As cápsulas são invólucros e são geralmente as mais produzidas dentro do ambiente magistral, requerendo de muita atenção durante sua manipulação, uma vez que, podem ocorrer facilmente erros relacionados a cálculos ou ainda contaminação cruzada. $\mathrm{Na}$ sua elaboração, as mesmas apresentavam-se disponíveis em diferentes tamanhos e cores, sendo que os tamanhos poderiam variar, conforme o volume, estando disponíveis na farmácia as seguintes cápsulas: 00 (volume de até $0,95 \mathrm{~mL}$ ), 0 (volume de até $0,68 \mathrm{~mL}$ ), 1 (volume de até $0,50 \mathrm{~mL}$ ), 2 (volume de até $0,37 \mathrm{~mL}$ ) e 3 (volume de até 0,30 $\mathrm{mL}) .9-11$

Após a pesagem e seleção do tamanho da cápsula, a quantidade de cápsulas específicas necessárias para comportar todo o pó eram adicionadas a uma encapsuladora. Inicialmente organizava-se a encapsuladora apropriada para cada tamanho de cápsula, abria-se a cápsula e adicionavase o corpo da mesma nos orifícios da encapsuladora, enquanto que a tampa era separada sobre a bancada. Logo após, adicionava-se o pó na encapsuladora, permitindo que o mesmo adentrasse no interior do corpo das cápsulas, sendo que para auxiliar nesse processo, utilizava-se espátula, socador e pequenos impactos mecânicos eram efetuados na encapsuladora contra a bancada para permitir a compactação do pó. Depois de se constatar que todo o pó encontrava-se dentro das cápsulas, conectava-se a tampa ao corpo, seguida do pré-fechamento, fechamento e limpeza. 
Logo após, 20 cápsulas eram selecionadas aleatoriamente para verificação do peso médio e desvio padrão, em que a variação dependia do peso médio das cápsulas. De acordo com Brasil. Agência Nacional de Vigilância Sanitária (2010)12, cápsulas duras e moles que tenham peso médio menor de 300 $\mathrm{mg}$, podem apresentar variações de até $\pm 10 \%$, enquanto que para aquelas que apresentam $300 \mathrm{mg}$ ou mais podem ter variações de até $\pm 7,5 \%$. Depois de o peso médio ser aprovado, as cápsulas eram devidamente armazenadas e o recipiente era previamente identificado com dados do paciente, do farmacêutico e do estabelecimento, assim como, do medicamento que foi manipulado. Por fim, as atividades no setor eram concluídas a partir da limpeza do material sujo, bancadas e equipamentos (Figura 1).

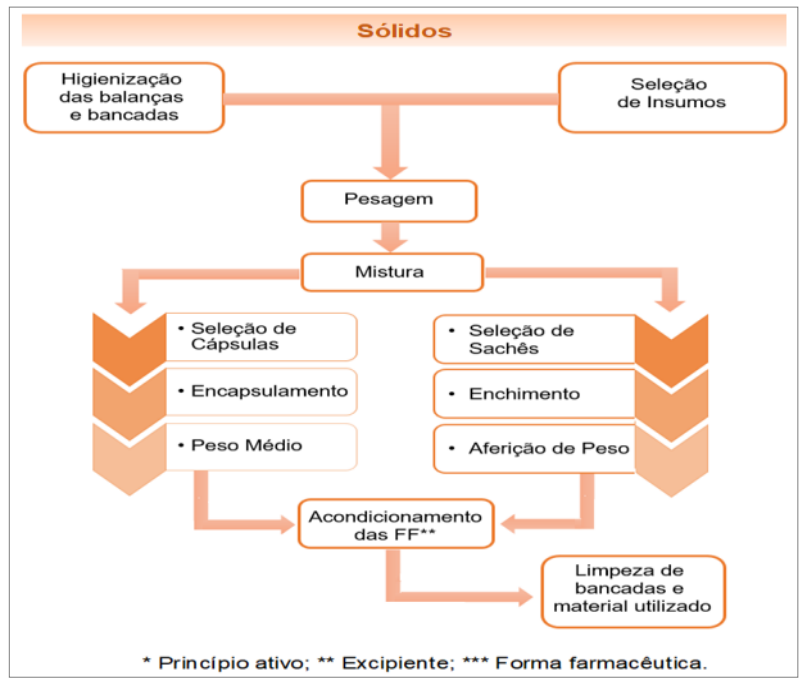

Figura 1: Fluxo das atividades realizadas no setor de sólidos (Fonte: Dados da Pesquisa).

\section{- Líquidos e semissólidos}

$\mathrm{O}$ setor de líquidos e semissólidos era composto por bancadas, armários com gavetas, pia, béqueres, bastões de vidro, almofarizes e pistilos, vidros de relógio, provetas, balanças analítica e semianalítica, dentre outros.

Dentre as principais preparações que eram feitas neste setor, podia-se destacar principalmente cremes, pomadas, suspensões, emulsões e xaropes ${ }^{13}$. Sendo que maior parte dos produtos manipulados e comercializados eram utilizados para o cuidado da pele e cabelo, como hidratantes, clareadores, protetor solar e condicionadores e xampus antiqueda. No momento da manipulação propriamente dita, inicialmente fazia-se a higienização da balança e da bancada e selecionava-se todos os princípios ativos e excipientes que seriam utilizados no momento da manipulação. Após selecionar os insumos farmacêuticos, estes eram organizados de forma sequencial, levando em consideração a ordem na qual seriam utilizados na manipulação. Em seguida, dados relacionados ao fornecer, validade e lote eram anotados na ficha do cliente, na determinada solicitação do produto.

$\mathrm{Na}$ sequência procedia-se à pesagem dos insumos e iniciava-se a manipulação propriamente dita, havendo primeiramente a adição de pós ou outros sólidos no gral, que depois de pulverizados, eram adicionados aos componentes semissólidos e líquidos que iriam compor a forma farmacêutica, homogeneizava-se e observava-se a consistência. Em algumas situações essências eram adicionadas, sobretudo, em xampus e hidratantes. Por fim, os produtos eram envasados e o rótulo era adicionado ao recipiente, contendo as mesmas informações dos sólidos e havia-se a limpeza da balança e bancadas para iniciar uma nova manipulação (Figura 2).

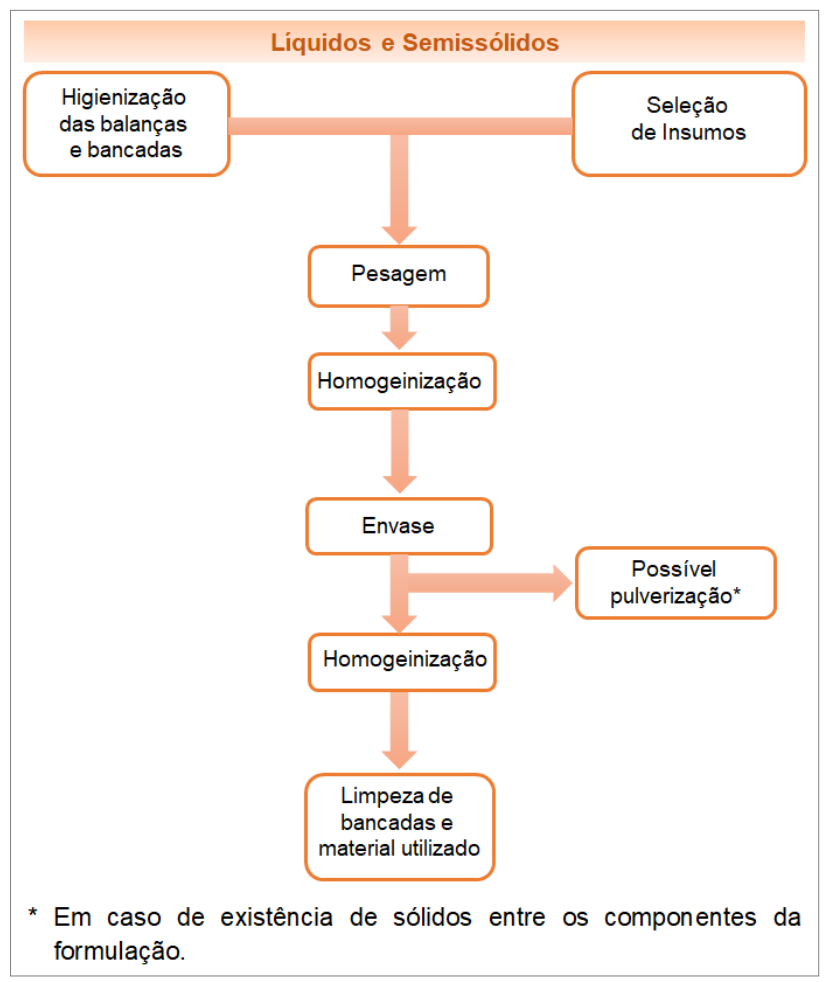

Figura 2: Fluxo das atividades realizadas no setor de semissólidos e líquidos (Fonte: Dados da Pesquisa).

\section{- Orientação farmacêutica}

A farmacêutica responsável encontrava-se em um ambiente mais reservado ao lado da recepção, em que neste momento se encarregava tanto de questões burocráticas quanto de possíveis atendimentos aos pacientes, que podia ser realizado pessoalmente ou por telefone, havendo a escuta ativa e suporte no momento da dispensação do medicamento 14 em que as principais dúvidas eram acerca da forma correta de uso, armazenamento e outros cuidados.

Entretanto, quando a farmacêutica responsável encontrava-se no ambiente de manipulação, o atendimento ainda sim era viável, contudo era feito por meio de telefones internos, um no setor de sólidos e outro no setor de líquidos e semissólidos, que se encontravam conectados a recepção, permitindo, portanto, mesmo que de forma mais restrita, a comunicação com o paciente. 


\section{- Outras atividades realizadas pelo estagiário}

A calibração é um processo de extrema importância, uma vez que foi possível verificar erros de medição e incerteza ${ }^{15}$ permitindo, portanto, saber se o equipamento em questão se encontra apto ao uso. Dessa forma, sempre deveria haver a calibração das balanças durante $o$ estágio que eram prioritariamente realizadas pelo estagiário, todos os dias antes do início das atividades laborais. Tal atividade era feita a partir da utilização de diferentes pesos, em que em cada dia utilizava-se um peso distinto, adicionando-o a balança três vezes e a partir daí a média era obtida. Após as balanças estarem aptas ao uso, iniciava-se o desenvolvimento de atividades do estágio propriamente dita. Ademais, o estagiário também era responsável por produzir o álcool a $70 \%$ para a assepsia de objetos e bancadas 16,17 em que, observava-se a quantidade de álcool que se necessitava, sendo o álcool a $96^{\circ}$ GL (Gay Lussac) usado para produzir o álcool a 70\%, empregando-se a seguinte fórmula:

$$
C 1 * V 1=C 2 * V 2
$$

- Percepção do estagiário frente as experiências vivenciadas

O estágio sem dúvidas me permitiu adquirir mais conhecimento teórico-prático acerca da manipulação de medicamentos, possibilitando observar que o farmacêutico na manipulação deve preocupar-se não somente com os produtos, mas também com a limpeza do ambiente de manipulação, $o$ atendimento aos clientes, fazer orientação e ainda ocupar-se de questões burocráticas relacionadas a gastos, fornecedores e a gestão da farmácia propriamente dita. Dessa forma, faz-se necessário ressaltar a importância desse estágio para o futuro farmacêutico, uma vez que, faz com que o mesmo possa experienciar o desenvolvimento de novas formulações, ter contato interpessoal com outros funcionários e também compreender a complexidade do funcionamento de uma farmácia magistral.

\section{CONCLUSÃO}

Durante o estágio em farmácia de manipulação, foi possível desenvolver atividades relacionadas à manipulação de formas farmacêuticas líquidas, sólidas e semissólidas. Ademais, para o futuro farmacêutico, a experiência de realizar estágio em farmácia de manipulação pode contribui para o desenvolvimento de novas habilidades e conhecimentos. Por fim, este tudo pode servir para nortear outros trabalhos que tenham o mesmo foco como centralizador, ou ainda, servir como forma de inspirar os estudantes de farmácia a conhecer mais profundamente a área de manipulação de medicamentos.

\section{REFERÊNCIAS}

1. UFCG. Universidade Federal de Campina Grande. Resolução nº8/2009. 2009. Disponível em: <http://www.ufcg.edu.br/ costa/resolucoes/res_16 082009.pdf>. Acesso em: 16 nov. 2018.

2. CFF. Conselho Federal de Farmácia. Guia prático do farmacêutico magistral. Brasília: CFF; 2017.

3. BRASIL. Agência Nacional de Vigilância Sanitária. Resolução da diretoria colegiada - RDC $\mathrm{n}^{\mathrm{o}}$ 67, de 8 de outubro de 2007. 2007. Disponível em:<

https://www20.anvisa.gov.br/segurancadopaciente /index.php/legislacao/item/rdc-67-de-8-deoutubro-de-2007 >. Acesso em: 05 fev. 2019.

4. Busanello C, Gomes E, Galina KJ, Gamara Júnior JS, Provasi M, Hashimoto MSM, Hoffmann RAG. Farmácia com manipulação: guia da profissão farmacêutica. Curitiba: CRF- PR; 2017.

5. Alves AP, Moura A, Neutgem ERV, Silva JM, Cunha NS, Oka SK et al. Avaliação das boas práticas de manipulação nas farmácias com manipulação de Cuiabá e Várzea Grande, Estado de Mato Grosso. Rev Bras Farm. 2009;90(1):75-80.

6. Santos DCFS. Revisão da qualidade na farmácia magistral. 2013. Disponível em: < http://www.anfarmag.com.br/files/artigotecnico/20130725_111837_02519.pdf>. Acesso em: 17 nov. 2018.

7. Andrade Júnior FP, Barbosa VSA. Monitoria acadêmica em parasitologia humana: um relato de experiência. Rev Saúde Com. 2017;13(3):972-75.

8. Neves HCC, Souza ACS, Medeiros M, Munari DB, Ribeiro LCM, Tipple AFV. Segurança dos trabalhadores de enfermagem e fatores determinantes para adesão aos equipamentos de proteção individual. Rev Latino-Am Enfermagem. 2011;19(2):354-61.

9. Maia Neto JF. Farmácia hospitalar e suas interfaces com a saúde. Editora RX: São Paulo; 2005

10. SBRT. Serviço Brasileiro de Respostas Técnicas. Dossiê técnico - Manipulação de cápsulas. 2012. Disponível $<$ http://www.respostatecnica.org.br/dossietecnico/downloadsDT/NjEwOQ $>$. Acesso em: 20 nov. 2018.

11. Allen Júnior LV. Introdução à farmácia de Remington. Artmed: Porto Alegre; 2016.

12. BRASIL. Agência Nacional de Vigilância Sanitária. Farmacopeia Brasileira. Brasília: Anvisa; 2010.

13. USP. Universidade de São Paulo. Formas Farmacêuticas. 2016. Disponível em: $<$ https://edisciplinas.usp.br/pluginfile.php/415259 3/mod_resource/content/9/FORMAS\%20FARMA CEUTICAS\%20aula\%20atual.pdf>. Acesso em: 30 nov. 2018.

14. Angonesi D, Rennó MUP. Dispensação farmacêutica: proposta de um modelo para a prática. Ciênc saúde coletiva. 2011;16(9):3883-91.

15. IPEM-PR. Instituto de Pesos e Medidas do Estado 
do Paraná. Calibração de Equipamentos. 2014. Disponível em:

http://www.saude.pr.gov.br/arquivos/File/apresent acao_calibracao_de_equipamentos_2014.pdf>. Acesso em: 06 dez. 2018.

16. Tiyo R, Torquato AS, Jacques FO, Colombo TC. Determinação do álcool $70 \%$ utilizado para antissepsia em drogarias e farmácia de MaringáParaná. Rev Bras Farm. 2009;90(3):231-35.

17. Ribeiro MM, Neumann VA, Padoveze MC, Graziano KU. Eficácia e efetividade do álcool na desinfecção de materiais semicríticos: revisão sistemática. Rev Latino-Am Enfermagem. 2015;23(4):741-52.

\section{CONFLITO DE INTERESSES}

Os autores declaram não haver conflitos de interesse.

\section{AUTOR PARA CORRESPONDÊNCIA}

Francisco Patricio de Andrade Júnior

R. Tab. Stanislau Eloy, 41 - Conj. Pres. Castelo Branco III, 58050-585 João Pessoa - PB, Brasil

Email: juniorfarmacia.ufcg@ outlook.com

Submetido em 19/04/2019

Aceito em 28/08/2020 